\title{
NUESTRA EXPERIENCIA EN EL TRATAMIENTO DE LA INCONTINENCIA URINARIA DE ESFUERZO MASCULINA MEDIANTE CABESTRILLO SUBURETRAL REGULABLE TIPO REMEEX
}

\author{
Pedro Navalón Verdejo, Yoni Pallás Costa, Felipe Ordoño Domínguez, Elisa Monllor Peidro, \\ Joaquín Juan Escudero, Macarena Ramos de Campos y Francisco Sánchez Ballester.
}

Unidad de Urodinámica. Servicio de Urología. Hospital Universitario Casa de Salud. Universidad Católica de Valencia. Valencia. España.

\begin{abstract}
Resumen.- OBJETIVO: Poner de manifiesto nuestra experiencia en el tratamiento de la incontinencia urinaria de esfuerzo postprostatectomía con el sistema Remeex masculino (SRM). Asimismo, describimos los elementos que componen dicho sistema, sus indicaciones terapéuticas, técnica de implantación y su adecuado ajuste.
\end{abstract}

MÉTODO: Desde marzo de 2007 hasta enero de 2009 hemos intervenido a cinco varones afectos de incontinencia urinaria de esfuerzo postprostatectomía con edades comprendidas entre 57 y 71 años Imedia 66

\section{CORRESPONDENCIA}

Pedro Navalón Verdejo

Paseo Alameda, 38 - 2

46023 Valencia (España)

pedronavalon@yahoo.com

Aceptado para publicar: 12 de marzo 2010. años), mediante la colocación de una malla suburetral de tensión regulable tipo Remeex. Todos ellos presentaban una incontinencia urinaria severa con gran afectación de su calidad de vida y de un tiempo de evolución entre 2 y 10 años (media 3,5 años), precisando todos ellos entre 5 y 8 compresas diarias.

RESULTADOS: Todos los pacientes se mantienen secos en un tiempo de seguimiento medio de 15,4 meses (rango 6-28 meses), precisando ocasionalmente 2 de ellos compresa de seguridad si realizan ejercicio intenso. La puntuación del Incontinence Impact Questionnaire IIIQ 7) realizado antes de la intervención y a los 6 meses después, pasa de $68 \pm 7$ puntos a $10 \pm 3$, siendo el grado de satisfacción muy elevado.

CONCLUSIONES: EI SRM constituye una opción terapéutica válida para la incontinencia postprostatectomía, siendo una técnica reproducible, de fácil ejecución, que permite su reajuste mediante una pequeña incisión suprapúbica bajo anestesia local y en régimen ambulatorio estricto, con una baja tasa de complicaciones proporcionando unos resultados excelentes y duraderos.

Palabras clave: Cabestrillo suburetral ajustable. Incontinencia urinaria masculina. Remeex.

Summary.- OBJECTIVES: We show our experience in the treatment of post-prostatectomy stress urinary incontinence with the male Remeex system (MRS). We also describe the elements of the system, its therapeutic indications, technical implantation and appropriate adjustment. 
METHODS: From March 2007 to January 2009 five male patients aged 57 to 71 years (mean age 66 years) with post-prostatectomy stress urinary incontinence were operated on to insert a suburethral readjustable sling (Remeex). All patients had severe urinary incontinence with deterioration of their quality of life. The evolution period ran-ged from 2 to 10 years with an average of 3.5 years, requiring from 5 to 8 pads a day.

RESULTS: All patients are continent after a mean follow up of 15.4 months (range 6-28 months). Only two of them use one security pad when they perform physical efforts. The Incontinen-ce Impact Questionnaire (7) scores befo-re surgery and 6 months after diminished from $68 \pm 7$ to $10 \pm 3$. All patients are very satisfied.

CONCLUSIONS: MRS is a valid therapeutic option for post-prostatectomy incontinence, being a reproducible technique, of easy execution, that allows readjustment through a suprapubic incision under local anesthesia as an outpatient procedure. It has a low complication rate with excellent and endured results.

Keywords: Adjustable male sling. Male urinary incontinence. Remeex.

\section{INTRODUCCIÓN}

La etiología mas frecuente de incontinencia urinaria de esfuerzo en el varón es la lesión iatrogénica del sistema esfinteriano proximal, fundamentalmente tras cirugía que secciona o amplía el cuello vesical, ya sea mediante intervención por vía endoscópica o a cielo abierto. En todos estos pacientes, la continencia queda confinada al sistema esfinteriano distal, pero si éste también se lesiona, la incontinencia es inevitable (1).

El creciente empleo de la prostatectomía radical en el tratamiento del carcinoma prostático es el principal condicionante del actual incremento en el número de casos de incontinencia urinaria de esfuerzo masculina. No obstante, resulta difícil valorar la incidencia y prevalencia exactas de la incontinencia postprostatectomía, ya que existen grandes variaciones según las diversas series publicadas. Así, la incidencia de incontinencia de esfuerzo tras cirugía de hiperplasia benigna de próstata (HBP) se cifra en torno al 0 y $8 \%$, mientras que su aparición tras prostatectomía radical por adenocarcinoma prostático varía entre 0,5 al 50\% según diversos autores (24). Estas grandes variaciones en su incidencia se deben en gran medida a la ausencia de una definición universalmente aceptada que favorece el empleo de conceptos imprecisos como incontinencia leve, incon- tinencia socialmente aceptada, incontinencia total, etc. Además, los datos han sido recogidos de forma dispar: encuesta telefónica, entrevista médica, autocuestionarios..., con falta de uniformidad en la valoración de la incontinencia (3). Por otra parte, también es de gran importancia el tiempo transcurrido tras la cirugía en la evaluación de la incontinencia, aunque la mayoría de autores coinciden en que se debe valorar al menos un año después de la prostatectomía (3).

Las opciones terapéuticas de la IUE masculina son diversas, desde la realización de ejercicios Kegel para la rehabilitación del suelo pélvico, tratamiento farmacológico, electroestimulación, implante de balones parauretrales, etc...; no obstante, las únicas técnicas de las que se dispone resultados a largo plazo son las inyecciones periuretrales de diversas sustancias ocupantes de espacio (que suelen tener un elevado coste económico y proporcionan una continencia parcial y temporal en hombres) y la colocación de un esfínter artificial, con una tasa de éxitos cercana al $90 \%$, siendo considerada en la actualidad el patrón de referencia con el que se comparan todos los demás procedimientos, aunque no está exento de complicaciones, fallos y necesidad de revisiones quirúrgicas conforme pasa el tiempo $(5,6)$. En la última década, fundamentándose en los excelentes resultados obtenidos con las mallas suburetrales libres de tensión en la mujer, han surgido diversas técnicas de sling masculino con un seguimiento y experiencia breves. (7-10).

\section{PRESENTACIÓN}

El Sistema Remeex Masculino (SRM) consta de una malla suburetral de prolene monofilamento de $3^{\prime} 5 \times 1^{\prime} 5 \mathrm{~cm}$, cuyos extremos finalizan en una sutura festoneada de prolene $\mathrm{n}^{\circ} 1$. El regulador mecánico, llamado varitensor, constituye un implante subcutáneo permanente que se acopla sobre una placa base que descansa sobre la fascia de los rectos anteriores a unos $2 \mathrm{~cm}$ de la sínfisis del pubis. Un manipulador externo que sale a través de la herida suprapúbica permite ajustar la presión suburetral ejercida por la malla. Un destornillador especial, llamado desacoplador, permite desconectar y el manipulador del varitensor una vez que se haya obtenido el grado de continencia deseado, permitiendo así retirar el manipulador.

El varitensor es un pequeño dispositivo de 1 $\times 1 \times 2^{\prime} 5 \mathrm{~cm}$, con un eje interno que permite enrollar los hilos de tracción. Estos hilos se pasan a través de dos orificios laterales del varitensor, y emergen por un orificio central que tiene un tornillo que fija 
los hilos de ambos lados cuando este se atornilla. A modo de sistema de poleas, el varitensor permite administrar o restar tensión a la malla mediante giro a favor o en contra del sentido de las agujas del reloj.

\section{MATERIAL Y MÉTODOS}

Desde marzo de 2007 hasta enero de 2009 hemos intervenido a cinco varones afectos de incontinencia urinaria de esfuerzo con edades comprendidas entre 57 y 71 años (media 66 años), mediante la colocación de una malla suburetral de tensión regulable tipo Remeex. En todos los casos la etiología fue iatrogénica, en un paciente tras adenomectomía prostática transvesical y en los otros cuatro tras prostatectomía radical retropúbica. Todos ellos presentaban una incontinencia urinaria severa con gran afectación de su calidad de vida y de un tiempo medio de evolución de 3,5 años (rango 2 y 10 años), precisando entre 5 y 8 compresas diarias.

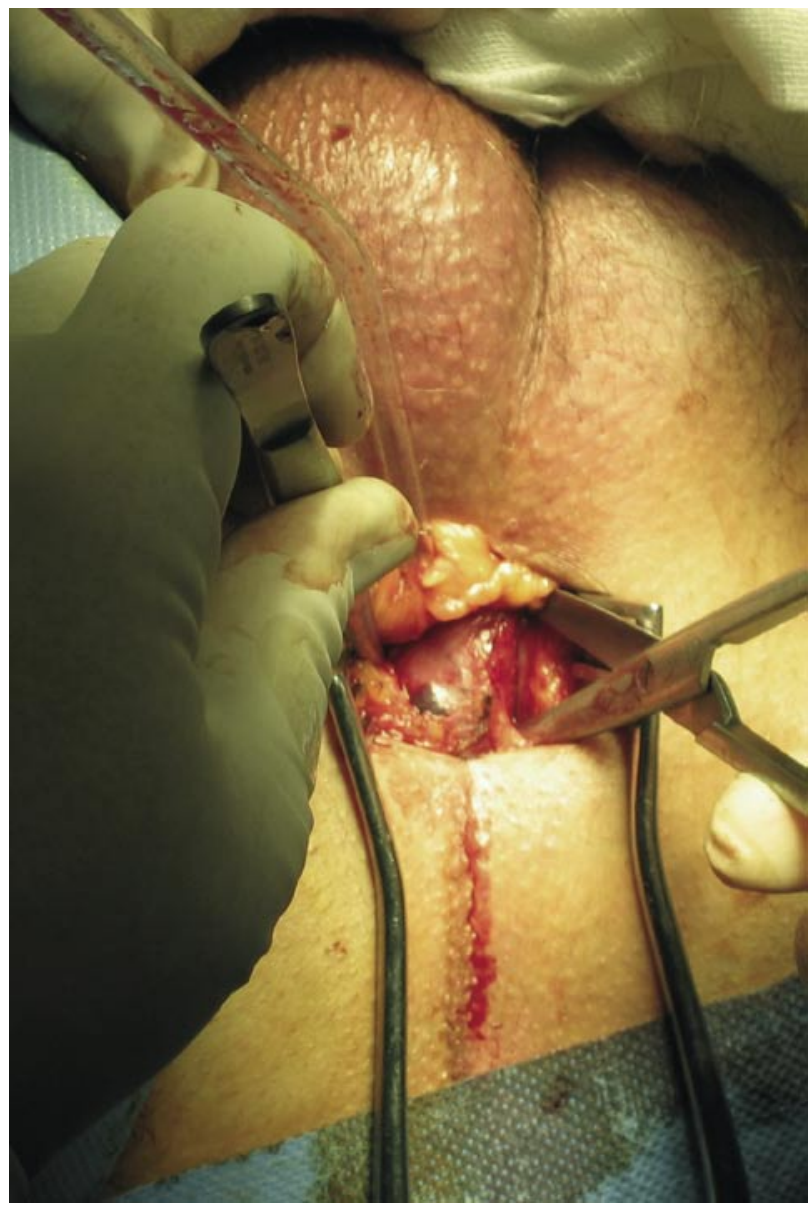

FIGURA 1. Disección y referencia de la uretra bulbar.
El estudio preoperatorio, además de poner de manifiesto la ausencia de progresión neoplásica en los casos de cáncer prostático, incluyó la realización de estudio urodinámico básico y uretrocistoscopia para descartar la existencia de obstrucción, inestabilidad vesical severa o vejiga de escasa capacidad que hubiesen sido considerados como criterios de exclusión para la realización del procedimiento. Los controles clínicos y de continencia se realizaron con periodicidad mensual durante los tres primeros meses y trimestral posteriormente. Para medir el impacto de la incontinencia sobre la calidad de vida, realizamos el Incontinence Impact Questionnaire (IIQ 7) en el preoperatorio y al menos a los 6 meses después de la intervención. Al mismo tiempo se efectúa estudio flujométrico y valoración ecográfica del residuo postmiccional.

Previa profilaxis antibiótica, la interveción se llevó a cabo bajo anestesia raquídea, con el pa-

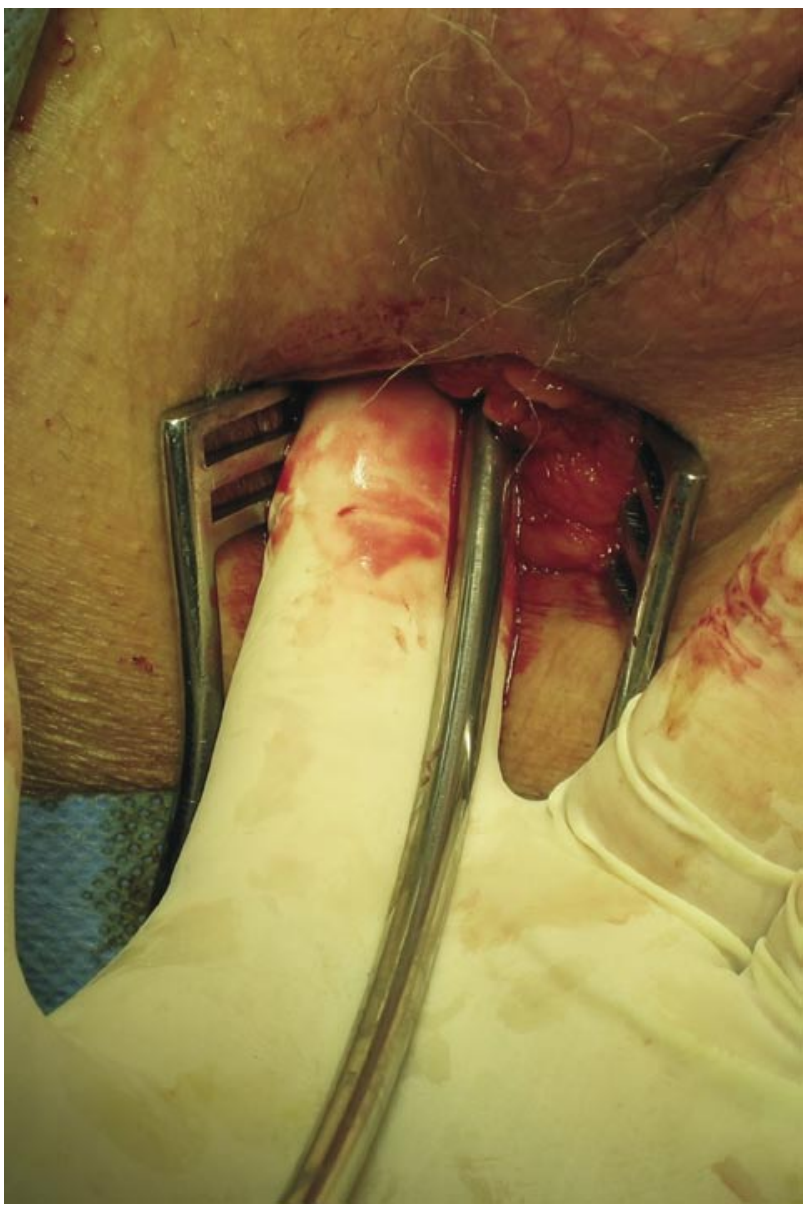

FIGURA 2. Inserción de la aguja en el espacio retropúbico. 

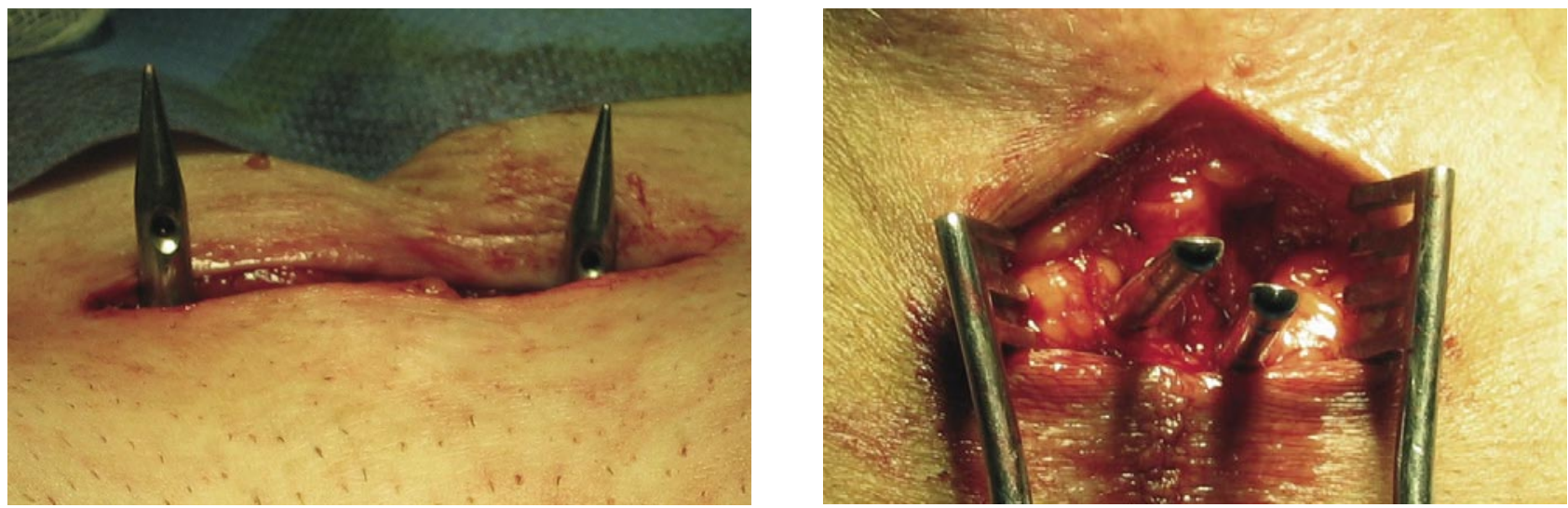

FIGURA 3. Posición de las agujas tras su inserción.

ciente en posición de litotomía con moderado Trendelemburg y elevación de la bolsa escrotal. En todos los pacientes se realizó el procedimiento mediante la siguiente secuencia operatoria:

\section{Colocación de sonda uretral Foley $n^{\circ} 18 \mathrm{Ch}$.}

2. Incisión trasversal suprapúbica de $4-5 \mathrm{~cm}$, disecando tejido subyacente hasta llegar a la aponeurosis de los rectos anteriores, creando un lecho supraaponeurótico para alojar el varitensor

3. Otra incisión perineal media longitudinal de 4-5 $\mathrm{cm}$, disecando tejido hasta localizar la uretra bulbar rodeada del músculo bulbocavernoso (Figura 1).

4. Disección de la rama isquiopúbica interna, abriendo la fascia del diafragma urogenital muy cerca del hueso, hasta que nos permita introducir la punta del dedo en el espacio retropúbico creado, haciendo una disección digital ascendente para disminuir todo lo que podamos la distancia entre la punta del dedo y la aponeurosis del recto anterior (Figura 2).

5. Punción en sentido perineo-abdominal ascendente mediante una aguja pasahilos tipo Stamey modificada de $20 \mathrm{~cm}$ y $60^{\circ}$ de ángulo, que se introduce dirigiendo su recorrido retropúbico con la punta del dedo y pasando la aguja rozando la cara interna del hueso procurando no lesionar vejiga ni uretra. Se repite la maniobra de punción en el lado contralateral (Figura 3).

6. Cistouretroscopia para confirmar la integridad uretrovesical.

7. Enhebrado de los hilos de tracción en las agujas y extracción de los mismos por la incisión suprapúbica, tirando de ambos extremos hasta que la malla de polipropileno quede en contacto con el músculo bulbocavernoso (Figura 4).

8. Fijación de la malla con puntos sueltos de material absorbible a nivel parauretral, procurando que quede totalmente extendida.

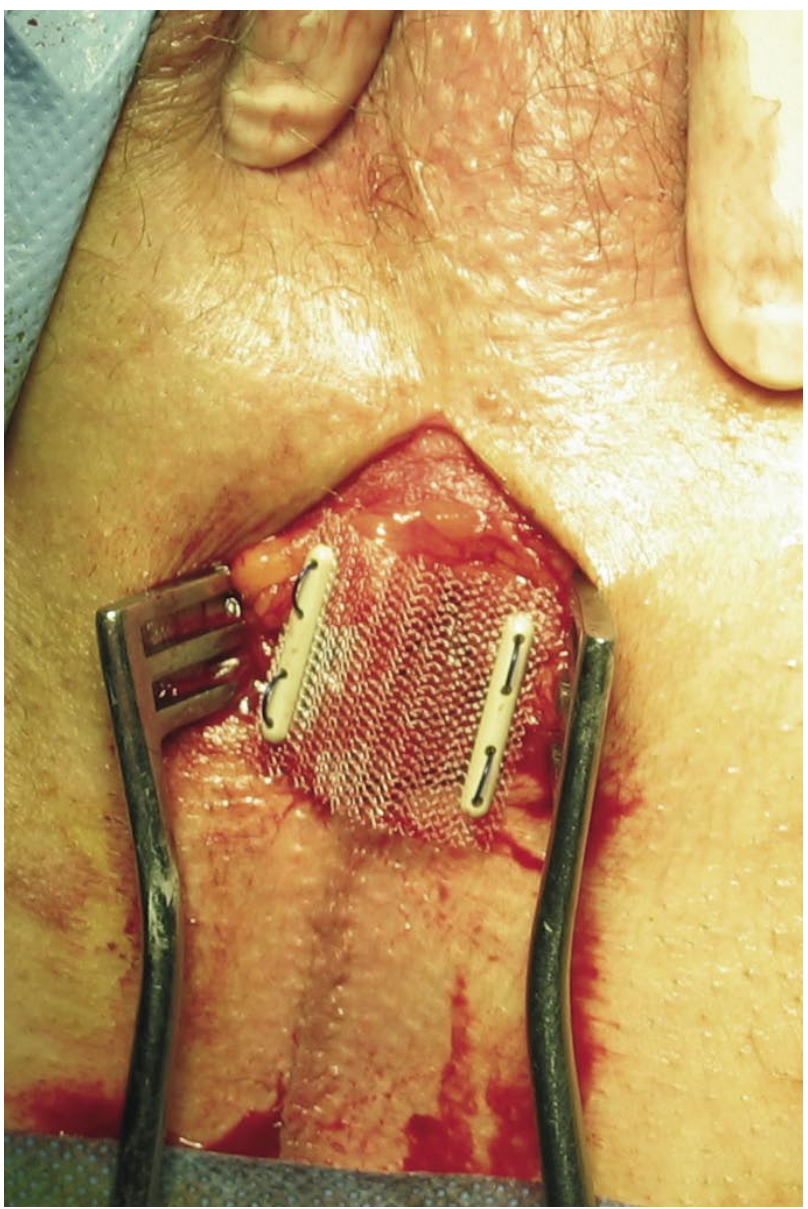

FIGURA 4. Colocación de la malla suburetral tras pasar los hilos. 
9. Cierre de la incisión perineal con material absorbible.

10. Enhebrado de los hilos de tracción en el varitensor a través de los correspondientes orificios laterales, emergiendo por el orificio central. Estiramos de ambos extremos hasta que el varitensor quede a una distancia de unos 4 traveses de dedo de la aponeurosis del recto anterior, fijando ambos extremos apretando el tornillo que posee el orificio de salida de los hilos, y cortando el hilo sobrante a ras de dicho tornillo (Figura 5).

11. Se gira el manipulador en sentido horario, enrollando así los hilos de tracción en el carrete del varitensor, el cual debe quedar suelto, sin ninguna tensión sobre placa base que descansa en la aponeurosis de los rectos anteriores (Figura 6).

12. La intervención finaliza cerrando la incisión suprapúbica, saliendo por el centro de la herida el

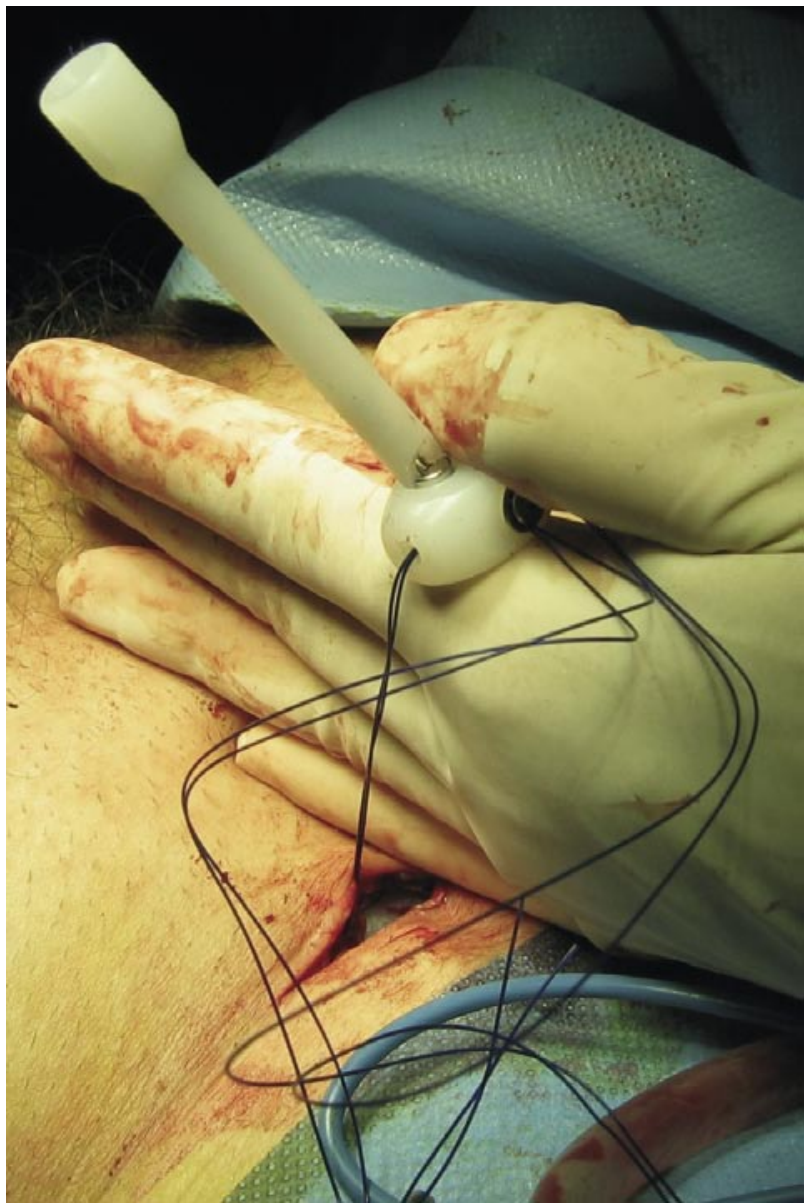

FIGURA 5. Fijación de los hilos del varitensor a cuatro traveses de dedo. extremo externo del manipulador, que permanece conectado al varitensor (Figura 7).

Si durante el procedimiento no hubo perforación vesical ni uretral, a las 24 horas siguientes a la intervención, se llena la vejiga con aproximadamente $300 \mathrm{cc}$ de salino fisiológico a través de la sonda previamente a su retirada y con el paciente en bipedestación, le invitamos a realizar maniobras de Valsalva (toser). Si se evidencia incontinencia, rotamos el manipulador en sentido horario hasta que los escapes cesen, comprobando la continencia habitualmente cada 4 giros completos. Cada vuelta completa del manipulador externo en sentido horario enrolla un milímetro de hilo en el carrete del varitensor, elevando el cabestrillo.

Una vez que el paciente ha vaciado espontáneamente la vejiga, comprobamos la ausencia de residuo postmiccional significativo y procedemos a desconectar el manipulador utilizando el desacopla-

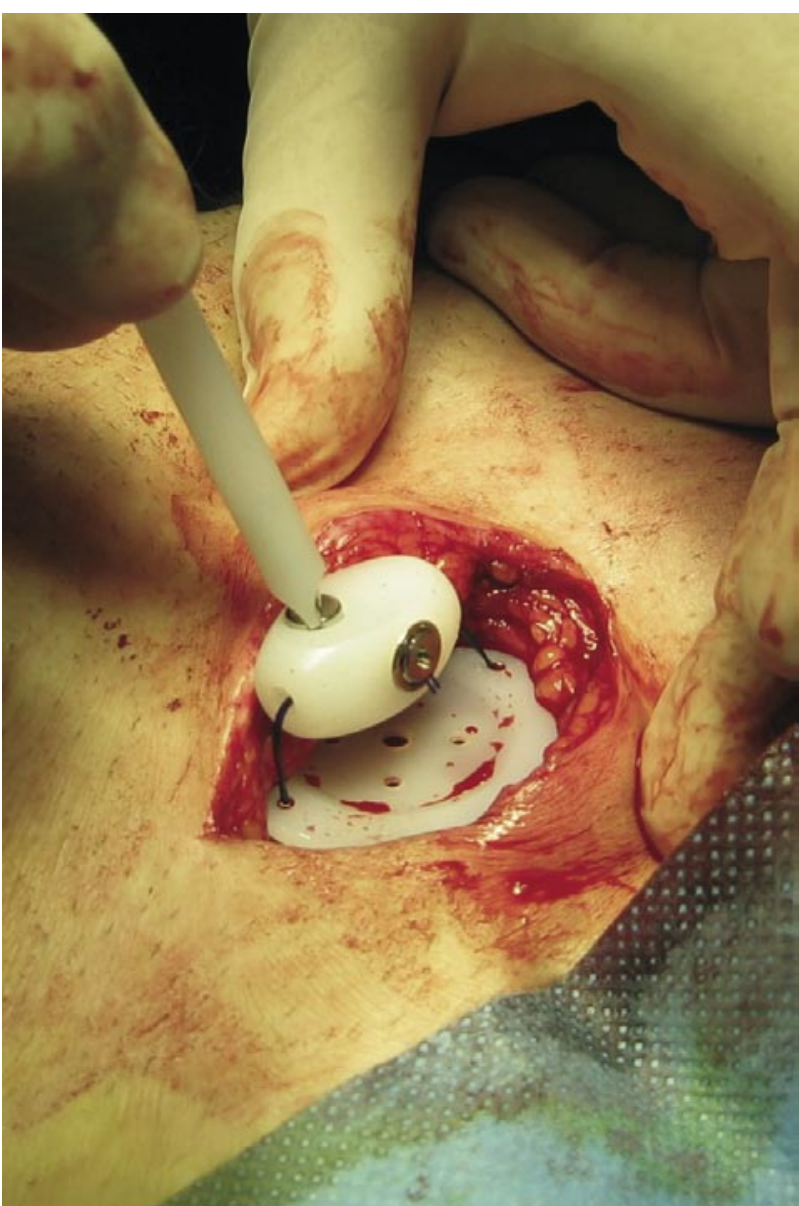

FIGURA 6. Enrollado del carrete del varitensor rotando el manipulador 
dor, el cual se rota un cuarto de vuelta y tiramos suavemente del manipulador hasta su extracción.

Si durante la cirugía hay lesión uretrovesical, se recomienda mantener la sonda y realizar el ajuste de la tensión a los 4 ó 5 días posteriores a la intervención.

En aquellos pacientes en los que reaparece la incontinencia tras haber sido dados de alta, hay que realizar un nuevo reajuste de la tensión de la malla volviendo a abrir únicamente el área suprapúbica bajo anestesia local, reacoplando el manipulador en el varitensor mediante el desacoplador, repitiendo el procedimiento del reajuste.

\section{RESULTADOS}

En todos los pacientes el procedimiento se realizó bajo anestesia raquídea siendo el tiempo operatorio inferior a 60 minutos. Las pérdidas hemáticas fueron inapreciables y en ningún caso se constató

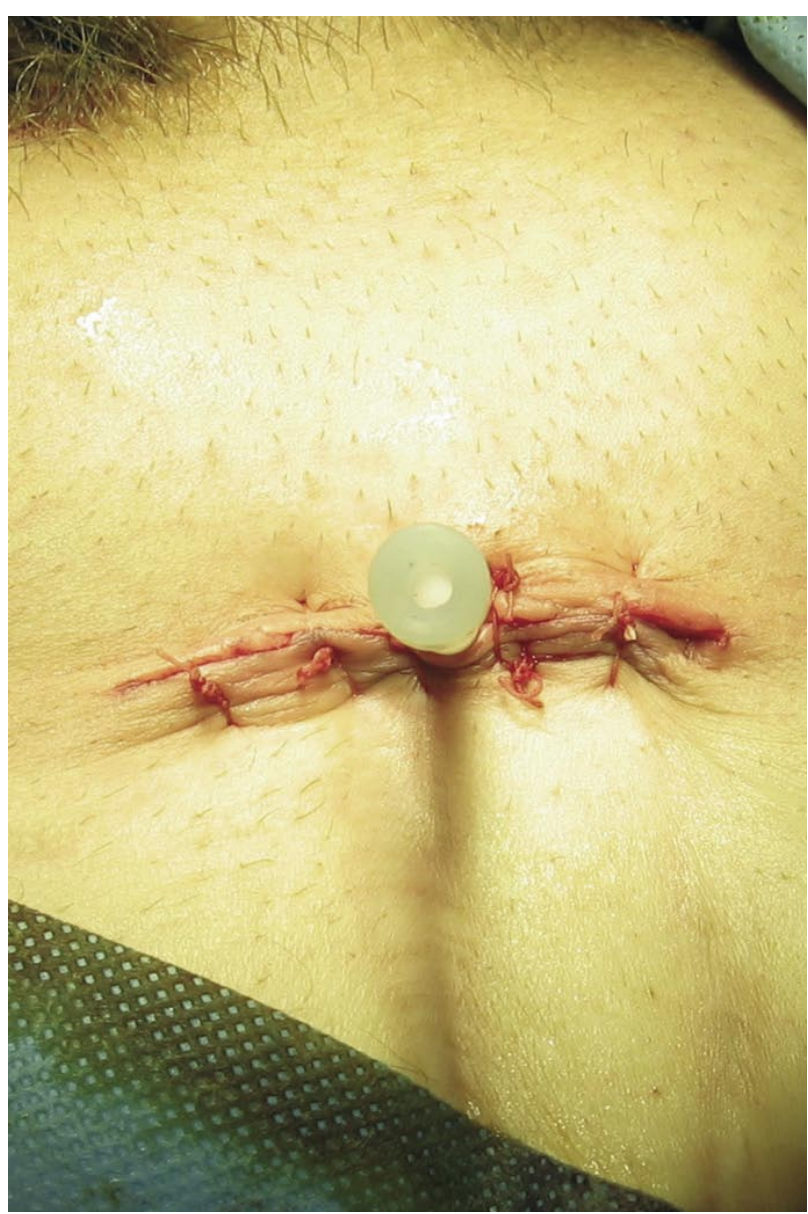

FIGURA 7. Posición final del manipulador previa a su extracción. lesión uretrovesical en la cistoscopia peroperatoria. El dolor postoperatorio se controló con antiinflamatorios no esteroideos (metamizol magnésico) y fueron dados de alta hospitalaria antes de las 24 horas posteriores a la intervención. No hubo retención urinaria en ningún caso ni infecciones de la herida y todos los pacientes se mantuvieron continentes durante los primeros meses. No obstante, cuatro de los cinco pacientes precisaron reajuste bajo anestesia local a los 3 ó 4 meses de la intervención por reaparición de incontinencia, en todos los casos de menor cuantía que antes de la colocación de la malla y dicho reajuste se realizó en régimen ambulatorio estricto.

El tiempo de seguimiento medio ha sido de 15,4 meses (rango 6-28). Todos los pacientes se mantienen secos, precisando ocasionalmente 2 de ellos compresa de seguridad si realizan ejercicio intenso.

El estudio flujométrico realizado a los 6 meses de la intervención evidenció un flujo miccional aceptable en todos los pacientes con un Qmax que osciló entre 15 y $19 \mathrm{ml} / \mathrm{s}$ (media 16'7 ml/s), con ausencia de residuo postmiccional significativo evaluado ecográficamente y ninguno de ellos refirió síntomas sugestivos de hiperactividad de novo.

La puntuación del Incontinence Impact Questionnaire (IIQ 7) realizado antes de la intervención y a los 6 meses después, pasa de $68 \pm 7$ puntos a $10 \pm 3$, siendo el grado de satisfacción muy elevado.

\section{DISCUSIÓN}

La IUE tras cirugía prostática constituye una desagradable y temida complicación, afortunadamente poco frecuente, pero de difícil solución, que provoca una merma importante en la calidad de vida del paciente que la padece y una gran frustración al médico que la provoca.

A lo largo de la historia se han empleado diversas técnicas para intentar corregirla, desde la utilización de pinzas de pene, cuyo uso prolongado produce dolor peneano y lesiones en la piel. Asimismo, la colocación de sondas acarrea el riesgo de infecciones frecuentes, formación de cálculos e incluso cambios metaplásicos y neoplásicos en la mucosa. Igualmente, los colectores externos tipo preservativo son de difícil manejo en pacientes prostatectomizados debidos al frecuente acortamiento del pene (11). También, las inyecciones transuretrales de colágeno o de otros polímeros así como los balones periuretrales han aportado unos resultados mediocres a mediolargo plazo, con unos índices de éxitos cifrados entre el 8 al $20 \%(12,13)$. 
El tratamiento que hasta la fecha ha demostrado proporcionar los mejores resultados a largo plazo es el esfínter artificial, con una tasa de éxitos que fluctúa entre el $59 \%$ y el $90 \%(14,15)$, constituyendo en la actualidad el patrón de referencia con el que se comparan todas las demás técnicas. No obstante, se trata de un artilugio que trata la incontinencia urinaria de forma muy poco fisiológica, ya que comprime toda la uretra y puede desarrollar erosión y atrofia de esta, requiriendo además un adiestramiento especializado por parte del paciente. Asimismo, el esfínter artificial no está exento de complicaciones, requiriendo ser retirado entre el $14 \%$ al $17 \%$ de los pacientes por erosión, infección o mal funcionamiento del sistema, precisando revisiones quirúrgicas en los cinco primeros años tras su colocación en el $17 \%$ al $57 \%$ de los casos $(14,16)$. Sea como fuere, el esfínter urinario artificial produce una importante morbi-mortalidad $(11,17)$, por lo que en los últimos años han surgido diferentes alternativas terapéuticas. En la búsqueda de estas alternativas, la colocación de una banda suburetral constituye un procedimiento mucho menos complejo, a lo que se suma la consecución de una micción más fisiológica y no requiere aprendizaje de dispositivo alguno. En este contexto, se han comercializado dos tipos distintos de procedimientos de "sling suburetral": el que se realiza con paso de agujas retropúbicas y el que recurre al anclaje de la malla a las ramas isquiopubianas.

Las primeras experiencias con mallas ancladas (Invance ${ }^{\circledR}$ AMS) son muy recientes, habiéndose cifrado unas tasas de continencia en torno al $80 \%$ en los casos de IUE leve-moderada y del $50 \%$ en los casos de IUE severa, aunque se trata de series muy pequeñas y con un tiempo de seguimiento corto 7 , $8,10,18)$. En todos los trabajos llama la atención la escasez de complicaciones postoperatorias, no se citan problemas de erosión uretral y el riesgo de osteomielitis parece mínimo. No obstante, estudios recientes realizados con estas mallas suburetrales ancladas al hueso han puesto de manifiesto que si la presión de la malla contra la uretra es alta, la tasa de éxitos es elevada, pero en un tercio de los casos hubo retención urinaria postoperatoria, precisando tratamiento por aparición de imperiosidad miccional de novo en el $20 \%$ como expresión de un patrón urinario obstructivo $(19,20)$, que se confirma en estudios urodinámicos objetivándose un descenso medio del Qmax de 6,5 ml/s. Sin embargo, otros autores que propugnan la fijación de la malla con menor tensión sobre la uretra han tenido que volver a tensionar la malla en el $22 \%$ de los casos como consecuencia de un elevado índice de fracasos (19).

Algo parecido ocurre con las mallas transobturadoras (Advance ${ }^{\circledR}$ AMS), propuestas por sus investigadores para incontinencias leves o moderadas, ya que la excesiva tensión de sus extremos para conseguir la continencia frecuentemente provoca obstrucción, mientras que una insuficiente tensión suele proporcionar unos resultados insatisfactorios o pocos duraderos $(21,22)$. Para obviar este inconveniente, en los últimos años se ha divulgado la utilización de bandas suburetrales de tensión regulable, de las que básicamente se han comercializado dos: el sistema Remeex ${ }^{\circledast}$ (de patente y fabricación española) y el sistema Argus $^{\circledR}$. Con ambos se han publicado excelentes resultados en estudios multicéntricos (19, 22), con unas tasas de curación o mejoría en torno al $85 \%$ con los dos tipos de cabestrillo. No obstante, con el segundo hubo un mayor número de pacientes con problemas de obstrucción $15 \%$ de casos con retención aguda de orina con Argus frente al 0\% del Remeex), lo que demuestra que el SRM permite una adecuada presión suburetral que evita el escape urinario, pero sin provocar obstrucción $(19,22)$.

\section{CONCLUSIÓN}

Aunque nuestra serie es breve, consideramos que el SRM constituye una opción terapéutica válida para la incontinencia postprostatectomía, siendo una técnica reproducible, de fácil ejecución, que permite su reajuste mediante una pequeña incisión suprapúbica bajo anestesia local y en régimen ambulatorio estricto, con una baja tasa de complicaciones, proporcionando unos excelentes y duraderos resultados. Asimismo, al contrario que con el esfínter urinario artificial, la micción es más fisiológica, comprimiendo solamente la cara ventral y parte de la lateral de la uretra y así evitando lesiones por isquemia, carece de complicados mecanismos que precisen ser repuestos con el tiempo, no requiere manipulación especial por parte del paciente, por lo que puede implantarse en sujetos con poca habilidad manual. Además, el coste económico es muy inferior al del esfínter.

\section{BIBLIOGRAFIA y LECTURAS RECOMENDADAS (*lectura de interés $y^{* *}$ lectura fundamental)}

1. Postius J, Castro D. Tratamiento quirúrgico de la incontinencia urinaria masculina. Clínicas Urológicas de la Complutense, 639-660, Servicio de Publicaciones UCM, Madrid, 2000.

2. Kahihara C, Ferreira U, Nardi R, Matheus W, Rodrigues-Netto JR. Intervención fisioterapéutica precoz vesus tardía para tratamiento de la incontinencia urinaria masculina pos-prostatectomía. Arch Esp Urol, 2006; 59: 773. 
*3. García Novas R, Fernandez E, Sanz E, Diez J. Incontinencia urinaria postprostatectomía. En: Castro D, Espuña M (Ed). Tratado de Incontinencia Urinaria2006; 263-321. Ed Egraf, S.A; Madrid.

4. Herschorn S, Boccon-Gibod L, Bosch JL. Surgical treatment of Urinary incontinence". En: Abrams P, Loury S, Wein A (Ed). First International Consultation on Urinary Incontinence. 691-729. Healt Publication Ltd; Plymout (UK). 1999.

5. Secin F, Martínez-Salamanca J, Eilber K. Eficacia limitada del uso de agente inyectable permanente en el tratamiento de la incontinencia urinaria de esfuerzo tras prostatectomía radical. Arch Esp Urol, 2005; 58: 431.

6. Rodriguez O, San Martín A, Jalon A, Gonzalez rc, Alvarez M, Férnández JM, et al. Tratamiento de la incontinencia urinaria mediante sustancias inyectables. Análisis de nuestra serie. Arch Esp Urol, 2005; 58: 227.

7. Comiter CV. The male sling for stress urinary incontinence: a prospective study. J Urol, 2002; 167: 597.

*8. Queipo JA, Chicote F, Borrell A, Beltran JF, Garcia L, Pastor F. Tratamiento de la incontinencia urinaria de esfuerzo post-prostatectomía radical mediante malla anclada a ramas isquiopubianas". Actas Urol Esp 2005; 29: 764.

9. Clemens JQ, Bushman W, Schaeffer AJ. Questionnaire based assessment and urodynamic evaluation following bulbourethral sling procedure. $\mathbf{J}$ Urol, 1998; 159: 120.

10. Ullrich NF, Comiter CV. The male sling for stress urinary incontinence: urodynamic and subjective assenssment . J Urol, 2004; 172: 204.

11. Sandoval JC, Salazar A, Oyanedel P, Verdugo F, Romano S. Sling bulbouretral para la incontinencia urinaria de esfuerzo masculina post cirugía prostática. Rev Chil Urol, 2007; 72: 185.

12. Smith DN, Appell RA, Rackley RR, Winters JC. Collagen injection therapy for post-prostatectomy incontinence. J Urol, 1998; 160: 364.
13. Cansino J, Álvarez M, Martín M, Cabrera P, Pérez-Utrilla $\mathrm{M}$, Rodríguez $\mathrm{F}$, et al. Implante de balones parauretrales como tratamiento de la incontinencia urinaria masculina. Experiencia del Hospital Universitario la Paz". Arch Esp Urol, 2007; 60: 647.

14. Litwiller SE, Kim KB, Fone PD. Postprostatectomy incontienence and the artificial urinary sphincter : a long-term study of patient satisfaction and criteria for success. J Urol, 1996; 156: 1975.

15. Kudnetsov DD, Kim HL, Patel RV, Steimberg GD, Bales GT. Comparison of artificial urinary sphincter and collagen for the treatment of postprostatectomy incontinence. Urol, 2000; 56: 600.

16. Batista J, Arañó P, Errando C. Esfínter artificial en incontinencia urinaria severa: nueve años de experiencia. Arch Esp Urol, 2000; 53: 409.

17. Cerqueira M, Xambre L, Silva V, Santos R, Lages $\mathrm{R}$, Prisco R, et al. Sling bulbouretral. Experiencia del Servicio. Actas Urol Esp 2005; 29: 401.

18. Dikranian AH, Chang JH, Rhee EY, Aboseif SR. The male perineal sling : comparison of sling materials. J Urol, 2004; 172: 608.

**19. Sousa A, Cabrera J, Montovani F, Moretti M, Ioanidis E, Kondelidis N, et al. Adjustable suburethral sling (Male Remeex System ${ }^{\circledR}$ ) in the treatment of male stress urinary incontinence : a multicentric european study. European Urol, 2007; 52: 1473.

20. Franco N. Suburethral sling for male urinary incontinence. Infect Urol, 2001; 14: 10.

21. Moreno J, Romano SV, Galante I, Barrera J, Salinas J, Silmi A. Nuevo sling masculino Argus para el tratamiento de la incontinencia urinaria de esfuerzo. Arch Esp Urol, 2006; 59: 607.

**22. Romano SV, Metrebian SE, Vaz F, Muller V, D'ancona CA, Sousa A, et al. Resultados a largo plazo del estudio multicéntrico fase III del tratamiento de la incontinencia de orina post prostatectomía con un sling masculino ajustable: seguimiento mínimo 3 años. Actas Urol Esp, 2009; 33 : 309. 